\title{
SOME LIMITING PROPERTIES OF THE BOUNDS OF THE PRESENT VALUE FUNCTION OF A LIFE INSURANCE PORTFOLIO
}

\author{
YI ZHANG *** AND \\ ZHENGYAN LIN, ${ }^{* * * *}$ Zhejiang University \\ CHENGGUO WENG, ${ }^{* * * *}$ University of Waterloo
}

\begin{abstract}
Under certain assumptions on the dependence structure of the residual lives of the insured (i.e. their independence, positive association, or negative association), in this paper we establish some laws of large numbers for the convex upper bounds, derived by the technique of comonotonicity, of the present value function of a homogeneous portfolio composed of the whole-life insurance policies.
\end{abstract}

Keywords: Convex order; comonotonicity; present value function; Black-Scholes model; law of large numbers

2000 Mathematics Subject Classification: Primary 62P05

Secondary 91B30; 60F15

\section{Introduction}

In this paper we consider a homogeneous portfolio composed of $n$ whole-life insurance policies. All the insured are assumed to be aged $x$. We denote the residual life of the insured $l$ as $T_{l}, l=1, \ldots, n$, and assume that the $T_{l}$ have a common distribution function $F(x)$. We assume that the policy provides the insured $l$ with the benefit amount of $K_{l}$ units at the instant of death, i.e. at time $T_{l}$. Moreover, we assume that the interest accumulation function, $y(t)$, is appropriately modelled by the Black-Scholes model, as follows:

$$
y(t):=\delta t+\sigma B(t), \quad 0 \leq t \leq \infty .
$$

Here $\delta>0$ and $\sigma>0$ are constants and $B(t)$ is the standard Brownian process. Consequently, the present value of this homogeneous life portfolio is

$$
S_{n}:=\sum_{l=1}^{n} K_{l} \mathrm{e}^{-y\left(T_{l}\right)},
$$

where $T_{l}$ is assumed to be independent of the Brownian process $B(t)$ for every $l \in\{1, \ldots, n\}$. It is noteworthy that Ahcan et al. (2006) considered a model similar to model (1.1) and presented the convex upper and lower bounds for $S_{n}$. However, in Ahcan et al. (2006), $T_{l}$ in $S_{n}$ was taken to be $l$, not a random variable.

Received 5 October 2005; revision received 24 August 2006.

* Postal address: Department of Mathematics, Zhejiang University, Hangzhou, 310027, P. R. China.

** Partially supported by the NSFC, grant number 10471126 .

*** Partially supported by the NSFC, grant number 10571159, and the FDPHE, grant number 20060335032.

**** Postal address: Department of Statistics and Actuarial Science, University of Waterloo, Waterloo, Ontario N2L 3G1, Canada. Email address: wengcg@ hotmail.com 
As reflected in (1.1), recently the effects of stochastic interest rates have been emphasized in the life insurance literature because "durations of contracts in life insurance and the life annuity business are typically very long (often 30 or even more years) and the financial and investment risk - unlike the mortality risk - cannot be diversified with an increase in the number of policies' (Hoedemakers et al. (2006)). In the actuarial literature there are many papers covering random interest rates. To summarize, two kinds of methods are used to study this problem.

In most papers where the first method is used, the authors restrict themselves to calculating the first two or three moments of the present value function. For example, Panjer and Bellhouse (1980) and Bellhouse and Panjer (1981) used autoregressive models of order one to compute moments of insurance and annuity functions. In Beekman and Fuelling (1993), the authors gave expressions for the mean and the standard deviation of the future life insurance payments for certain policies. The first three moments of homogeneous portfolios of life insurance and endowment policies were calculated in Parker (1994a), (1994b), and these results were generalized to heterogeneous portfolios in Parker (1997). Papers involving the calculation of the first few moments in stochastic interest rate frameworks include Boyle (1976), Waters (1978), Wilkie (1976), Dhaene (1989), Norberg (1990), and many others.

The second method is to use the comonotonicity technique to estimate the upper and lower bounds of the prevent values. This technique was proposed by Dhaene and Goovaerts (1996), (1997) and adopted by Wang and Dhaene (1998), Goovaerts and Redant (1999), Goovaerts and Dhaene (1999), Dhaene et al. (2002a), (2002b), Goovaerts et al. (2000), Simon et al. (2000), Vyncke et al. (2001), Kaas et al. (2000), (2001), Ahcan et al. (2006), and others.

In this paper we aim to study the limiting properties of the upper bounds of the present value of model (1.1), derived by the technique of comonotonicity, since an average insurer usually has a large number of homogeneous policies and the upper bounds are very informative and useful to the insurer in making conservative estimates about the risks and calculating premiums. However, it would clearly be difficult to discuss the limiting distributions of the upper bounds of model (1.1) under a general dependence structure for the $K_{l}$ or the $T_{l}$. Therefore, we consider the cases in which $T_{l}, l=1, \ldots, n$, are respectively independent, negatively associated, and positively associated random variables, and establish some laws of large numbers for each dependence structure. Note that, even if $\left\{K_{l}, l=1, \ldots, n\right\}$ and $\left\{T_{l}, l=1, \ldots, n\right\}$ are independent random series, it is still not technically easy to establish laws of large numbers for the present value $S_{n}$ directly. The concepts of negative association and positive association are frequently used in statistical science to describe the dependence structure of random variables, since they encompass a wide range of dependence structures. Of course, other dependence structures may be considered. In conclusion, in this paper we propose to combine the techniques of comonotonicity and limit theory to further study present values (or other values) in actuarial science.

The outline of the paper is as follows: in Section 2 the convex bounds of the present value function are presented, and in Section 3 we discuss some limiting properties of the upper convex bounds of the present value function.

\section{Convex bounds of the present value function}

In this section we focus on discussing the convex bounds of the random sum (1.1). We denote by $U$ and $V$ two mutually independent random variables uniformly distributed on [0, 1$]$. Moreover, let $\Lambda$ also be a random variable, independent of both $U$ and $V$. We now recall the definition of convex order. 
Definition 2.1. Let $X$ and $Y$ be two random variables with finite means. We say that $X$ is less than $Y$ in convex order (written $X \leq_{\mathrm{cx}} Y$ ) if $\mathrm{E}[f(X)] \leq \mathrm{E}[f(Y)]$ for all real convex functions $f$ for which the expectations exist.

The following lemma will play a critical role in the subsequent discussion.

Lemma 2.1. 1. Let $\boldsymbol{X}=\left(X_{1}, \ldots, X_{n}\right)$ be a random vector. Then

$$
\sum_{i=1}^{n} \mathrm{E}\left[X_{i} \mid \Lambda\right] \leq \mathrm{cx} \sum_{i=1}^{n} X_{i} \leq_{\mathrm{cx}} \sum_{i=1}^{n} F_{X_{i}}^{-1}(U)
$$

where $F_{X}^{-1}(p)=\inf \left\{x \in \mathbb{R}: F_{X}(x) \geq p\right\}$ and $F_{X}(t)$ is the distribution function of $X$.

2. Let $\boldsymbol{V}=\left(V_{1}, \ldots, V_{n}\right)$ and $\boldsymbol{W}=\left(W_{1}, \ldots, W_{n}\right)$ be nonnegative random vectors, and assume that $\boldsymbol{X}=\left(X_{1}, \ldots, X_{n}\right)$ is a random vector independent of both $\boldsymbol{V}$ and $\boldsymbol{W}$. If, for every $\left(x_{1}, \ldots, x_{n}\right)$, we have

$$
\sum_{i=1}^{n} x_{i} V_{i} \leq \mathrm{cx} \sum_{i=1}^{n} x_{i} W_{i}
$$

then the corresponding scalar products are convex ordered, i.e.

$$
\sum_{i=1}^{n} X_{i} V_{i} \leq \mathrm{cx} \sum_{i=1}^{n} X_{i} W_{i}
$$

Proof. We refer the reader to Kaas et al. (2000) for the proof of part 1, and to Ahcan et al. (2006) for the proof of part 2.

Dhaene et al. (2002b) considered the case in which $X_{i}=\mathrm{e}^{-Y_{i}}$ in part 1 of Lemma 2.1, where $\left(Y_{1}, \ldots, Y_{n}\right)$ constitutes a multivariate normal random vector with parameters $\mathrm{E}\left[Y_{i}\right]=\mu_{i}$, $\operatorname{var}\left(Y_{i}\right)=\sigma_{i}$ and $\operatorname{cov}\left(Y_{i}, Y_{j}\right)=\sigma_{i j}, i, j=1, \ldots, n$. Using Lemma 2.1, Dhaene et al. (2002b, Section 4) derived bounds for $\sum_{i=1}^{n} X_{i}$ by taking $\Lambda=\sum_{j=1}^{n} \exp \left(\mu_{j}+\frac{1}{2} \sigma_{j}^{2}\right) Y_{j}$, as in the following lemma.

Lemma 2.2. 1. Let $U$ be uniform on $[0,1]$ and independent of the random variable $\Lambda$. Then

$$
\sum_{i=1}^{n} \exp \left(-\mu_{i}+\frac{1}{2} \sigma_{i}^{2}\left(1-r_{i}^{2}\right)-\sigma_{i} r_{i} \Phi^{-1}(V)\right) \leq \mathrm{cx} \sum_{i=1}^{n} \mathrm{e}^{-Y_{i}} \leq_{\mathrm{cx}} \sum_{i=1}^{n} \mathrm{e}^{-\mu_{i}+\sigma_{i} \Phi^{-1}(U)},
$$

where

$$
r_{i}=\operatorname{corr}\left(Y_{i}, \Lambda\right)=\frac{\operatorname{cov}\left(Y_{i}, \Lambda\right)}{\sqrt{\operatorname{var}\left(Y_{i}\right) \operatorname{var}(\Lambda)}}=\frac{\sum_{j=1}^{n} \mathrm{e}^{\mu_{j}+(1 / 2) \sigma_{j}^{2}} \sigma_{i j}}{\sigma_{i} \sqrt{\sum_{1 \leq k, l \leq n} \exp \left(\mu_{k}+\mu_{l}+\frac{1}{2}\left(\sigma_{k}^{2}+\sigma_{l}^{2}\right)\right) \sigma_{k l}}} .
$$

Now consider model (1.1). Since $\mathrm{E}\left[-y\left(T_{i}\right) \mid T_{i}\right]=-\delta T_{i}$, we have $\operatorname{var}\left(-y\left(T_{i}\right) \mid T_{i}\right)=$ $\sigma^{2} T_{i}$ and $\operatorname{cov}\left(-y\left(T_{i}\right),-y\left(T_{j}\right)\right)=\sigma^{2} \min \left\{T_{i}, T_{j}\right\}, i, j=1, \ldots, n$. Using the conditional expectation technique, we know that Lemma 2.2 applies to $\sum_{i=1}^{n} \mathrm{e}^{-y\left(T_{i}\right)}$ with the replacements $\mu_{i}=-\delta T_{i}, \sigma_{i}=\sigma \sqrt{T_{i}}, \sigma_{i j}=\sigma^{2} \min \left\{T_{i}, T_{j}\right\}$, and $\Lambda=\sum_{i=1}^{n} \mathrm{e}^{-\mu_{i}-(1 / 2) \sigma_{i}^{2}}\left(-y\left(T_{i}\right)\right)$. This leads to the following proposition. 
Proposition 2.1. In model (1.1), we have

$$
\begin{aligned}
\sum_{i=1}^{n} \exp \left(-\delta T_{i}+\frac{1}{2} \sigma^{2} T_{i}\left(1-R_{i}^{2}\right)-\sigma \sqrt{T_{i}} R_{i} \Phi^{-1}(V)\right) & \leq{ }_{\mathrm{cx}} \sum_{i=1}^{n} \mathrm{e}^{-y\left(T_{i}\right)} \\
& \leq{ }_{\mathrm{cx}} \sum_{i=1}^{n} \exp \left(-\delta T_{i}+\sigma \sqrt{T_{i}} \Phi^{-1}(U)\right),
\end{aligned}
$$

where

$$
R_{i}=\frac{\sum_{j=1}^{n} \mathrm{e}^{-\delta T_{j}-(1 / 2) \sigma^{2} T_{j}} \min \left\{T_{i}, T_{j}\right\}}{\sqrt{T_{i} \sum_{1 \leq l, k \leq n}^{n} \exp \left(-\delta\left(T_{l}+T_{k}\right)-\frac{1}{2} \sigma^{2}\left(T_{l}+T_{k}\right)\right) \min \left\{T_{l}, T_{k}\right\}}} .
$$

For notational convenience, we henceforth write

$$
\begin{aligned}
S_{n}^{\mathrm{uu}} & =\sum_{i=1}^{n} F_{K_{i}}^{-1}(U) \exp \left(-\delta T_{i}+\sigma \sqrt{T_{i}} \Phi^{-1}(U)\right), \\
S_{n}^{\mathrm{u}} & =\sum_{i=1}^{n} K_{i} \exp \left(-\delta T_{i}+\sigma \sqrt{T_{i}} \Phi^{-1}(U)\right), \\
S_{n}^{\mathrm{l}} & =\sum_{i=1}^{n} K_{i} \exp \left(-\delta T_{i}+\frac{1}{2} \sigma^{2} T_{i}\left(1-R_{i}^{2}\right)-\sigma \sqrt{T_{i}} R_{i} \Phi^{-1}(V)\right), \\
S_{n}^{\mathrm{ll}}= & \sum_{i=1}^{n} \mathrm{E}\left[K_{i} \mid \Theta\right] \exp \left(-\delta T_{i}+\frac{1}{2} \sigma^{2} T_{i}\left(1-R_{i}^{2}\right)-\sigma \sqrt{T_{i}} R_{i} \Phi^{-1}(V)\right),
\end{aligned}
$$

where $\Theta$ is a random variable independent of $\Lambda$ and $y\left(T_{i}\right), i=1, \ldots, n$, but which may depend on $K_{i}, i=1, \ldots, n$.

Using Proposition 2.1 and repeatedly applying the result of part 2 of Lemma 2.1, we can derive the following proposition about the bounds of the present value function (1.1).

Proposition 2.2. In model (1.1), we have $S_{n}^{1 \mathrm{l}} \leq_{\mathrm{cx}} S_{n}^{\mathrm{l}} \leq_{\mathrm{cx}} S_{n} \leq_{\mathrm{cx}} S_{n}^{\mathrm{u}} \leq_{\mathrm{cx}} S_{n}^{\mathrm{uu}}$.

Remark 2.1. Note that if $\Lambda$ is chosen differently, we may obtain different bounds for the present value function (1.1). For example, if we take $\Lambda=\sum_{i=1}^{n} \lambda\left(T_{i}\right) B\left(T_{i}\right)$ with any function $\lambda(x): \mathbb{R}^{+} \rightarrow \mathbb{R}^{+}$, the lower bounds are $S_{n}^{1}$ and $S_{n}^{11}$ as above with $R_{i}$ as follows:

$$
R_{i}=\frac{\sum_{j=1}^{n} \lambda\left(T_{j}\right) \min \left\{T_{i}, T_{j}\right\}}{\sigma \sqrt{T_{i}} \sigma_{\Lambda}}, \quad \text { where } \quad \sigma_{\Lambda}^{2}=\sum_{j=1}^{n} \sum_{k=1}^{n} \lambda\left(T_{k}\right) \lambda\left(T_{j}\right) \min \left\{T_{k}, T_{j}\right\}
$$

\section{The limiting properties of the upper convex bounds}

From the expressions for the bounds we obtained in the previous section, we seems that the upper convex bounds are more mathematically tractable than the lower bounds. Moreover, for the reason given in Section 1, the insurer may be much more interested in the results of studying the former. In light of this, we now investigate the limiting properties of the upper convex bounds. Specifically, we establish laws of large numbers under three kinds of assumption on the dependence structure: independence, negative association, and positive association. 
Let

$$
\begin{aligned}
\mu^{\mathrm{uu}} & =F_{K_{1}}^{-1}(U) \mathrm{E}\left[\exp \left(-\delta T_{1}+\sigma \sqrt{T_{1}} \Phi^{-1}(V)\right) \mid V\right], \\
\mu^{\mathrm{u}} & =\mathrm{E}\left[K_{1}\right] \mathrm{E}\left[\exp \left(-\delta T_{1}+\sigma \sqrt{T_{1}} \Phi^{-1}(V)\right) \mid V\right] .
\end{aligned}
$$

The following theorem holds when the residual lives are independent.

Theorem 3.1. Consider model (1.1).

1. If $T_{l}, l=1, \ldots, n$, are independently and identically distributed random variables, then

$$
\frac{S_{n}^{\mathrm{uu}}}{n} \rightarrow \mu^{\mathrm{uu}} \text { almost surely, as } n \rightarrow \infty .
$$

2. If $K_{l}, l=1, \ldots, n$, are independently and identically distributed random variables, then

$$
\frac{S_{n}^{\mathrm{u}}}{n} \rightarrow \mu^{\mathrm{u}} \quad \text { almost surely, as } n \rightarrow \infty .
$$

Proof. To prove part 1, we let

$$
Y_{l}=F_{K_{l}}^{-1}(U) \exp \left(-\delta T_{l}+\sigma \sqrt{T_{l}} \Phi^{-1}(V)\right) \quad \text { and } \quad \mathcal{B}_{l}=\sigma\left(T_{1}, \ldots, T_{l}, U, V\right) .
$$

Then

$$
\left\{\sum_{l=1}^{n}\left(Y_{l}-\mathrm{E}\left[Y_{l} \mid \mathcal{B}_{l-1}\right]\right), \mathcal{B}_{n}, n=1,2, \ldots\right\}
$$

is a martingale and

$$
\begin{aligned}
\mathrm{E}\left[Y_{l} \mid \mathcal{B}_{l-1}\right] & =\mathrm{E}\left[F_{K_{l}}^{-1}(U) \exp \left(-\delta T_{l}+\sigma \sqrt{T_{l}} \Phi^{-1}(V)\right) \mid U, V\right] \\
& =F_{K_{1}}^{-1}(U) \mathrm{E}\left[\exp \left(-\delta T_{1}+\sigma \sqrt{T_{1}} \Phi^{-1}(V)\right) \mid V\right] \\
& =\mu^{\mathrm{uu}} .
\end{aligned}
$$

Hence, it follows from the strong law of large numbers for a martingale (see Hall and Heyde (1980, pp. 36-39)) that

$$
\frac{1}{n} \sum_{l=1}^{n}\left(Y_{l}-\mathrm{E}\left[Y_{l} \mid \mathcal{B}_{l-1}\right]\right) \rightarrow 0 \quad \text { almost surely, as } n \rightarrow \infty,
$$

which completes the proof of (3.1).

To prove part 2, we let

$Z_{l}=K_{l} \exp \left(-\delta T_{l}+\sigma \sqrt{T_{l}} \Phi^{-1}(V)\right) \quad$ and $\quad W_{l}=\mathrm{E}\left[K_{l}\right] \mathrm{E}\left[\exp \left(-\delta T_{l}+\sigma \sqrt{T_{l}} \Phi^{-1}(V)\right) \mid V\right]$.

Clearly $W_{l}=W_{1}=\mu^{\mathrm{u}}$ for all $l=1,2, \ldots$ Then, similarly to in the proof of (3.1), we have

$$
\frac{1}{n} \sum_{l=1}^{n}\left(Z_{l}-W_{l}\right) \rightarrow 0 \quad \text { almost surely, as } n \rightarrow \infty \text {. }
$$

That is,

$$
\frac{1}{n} \sum_{l=1}^{n} Z_{l} \rightarrow \mu^{\mathrm{u}} \quad \text { almost surely, as } n \rightarrow \infty .
$$

As a result, (3.2) follows immediately. 
Next we consider the respective cases in which $T_{l}, l=1, \ldots, n$, are mutually dependent in the following two senses.

Definition 3.1. 1. A random vector $\boldsymbol{X}=\left(X_{1}, \ldots, X_{m}\right)$ is said to be negatively associated if

$$
\operatorname{cov}\left(f\left(X_{i}, i \in L\right), g\left(X_{j}, j \in M\right)\right) \leq 0
$$

for all disjoint subsets $L$ and $M$ of $\{1, \ldots, m\}$ and all increasing functions $f: \mathbb{R}^{d(L)} \rightarrow \mathbb{R}$ and $g: \mathbb{R}^{d(M)} \rightarrow \mathbb{R}$, where $d(A)$ denotes the cardinality of the (generic) set $A$.

2. A random vector $\boldsymbol{X}=\left(X_{1}, \ldots, X_{m}\right)$ is said to be positively associated if

$$
\operatorname{cov}(f(\boldsymbol{X}), g(\boldsymbol{X})) \geq 0
$$

for all increasing functions $f, g: \mathbb{R}^{m} \rightarrow \mathbb{R}$.

For our further discussion, we introduce the following definition and present three lemmas. See Newman (1980) for the proof of Lemma 3.1, Lin (2003) for the proof of Lemma 3.2, and respectively Liu et al. (1999) and Birkel (1988) for the proofs of parts 1 and 2 of Lemma 3.3.

Definition 3.2. A sequence of functions $\left\{g_{i}(\cdot, \cdot), i \geq 1\right\}$ is said to satisfy a uniform Lipschitz condition of order one if there exists a constant $B>0$ such that

$$
\left|g_{i}(x+u, y+v)-g_{i}(x, y)\right| \leq B(|u|+|v|)
$$

for any $x, y, u, v \in \mathbb{R}$.

Lemma 3.1. If $X$ and $Y$ are positively associated random variables then, for any $s, t \in \mathbb{R}$,

$$
\left|\mathrm{E}\left[\mathrm{e}^{\mathrm{i} s X+\mathrm{i} t Y}\right]-\mathrm{E}\left[\mathrm{e}^{\mathrm{i} s X}\right] \mathrm{E}\left[\mathrm{e}^{\mathrm{i} t Y}\right]\right| \leq s t|\operatorname{cov}(X, Y)|,
$$

where $\mathrm{i}$ is the imaginary unit.

Lemma 3.2. Assume that $\left\{T_{i}, i \geq 1\right\}$ is a sequence of positively associated random variables with common density function $p(\cdot)$ and characteristic function $\varphi(\cdot)$. Let $p_{1 j}(\cdot, \cdot)$ and $\varphi_{1 j}(\cdot, \cdot)$ be the joint density function and the joint characteristic function of $T_{1}$ and $T_{j}, j \geq 2$, respectively. Then, if

(C1) $p(t)$ is bounded and satisfies a uniform Lipschitz condition of order one,

(C2) $p_{1 j}(\cdot, \cdot), j \geq 1$, satisfy a uniform Lipschitz condition of order one, and

(C3) $\varphi(\cdot)$ and $\varphi_{1 j}(\cdot, \cdot), j \geq 2$, are absolutely integrable,

it follows that, for any $D>0$ and $j \geq 2$,

$$
\sup _{x, y}\left|p_{1 j}(x, y)-p(x) p(y)\right| \leq \frac{1}{4 \pi^{2}} \int_{-D}^{D} \int_{-D}^{D}\left|\varphi_{1 j}(s, t)-\varphi(s) \varphi(t)\right| \mathrm{d} s \mathrm{~d} t+\frac{6 \sqrt{2} B(1+A)}{D},
$$

where $B$ is the constant implicit in condition (C2) and $A=\sup _{x} p(x)$.

Lemma 3.3. 1. Let $\left\{X_{i}, i \geq 1\right\}$ be a sequence of negatively associated random variables with a common distribution. For $0<p<2$, the condition necessary and sufficient to ensure that

$$
\frac{\sum_{i=1}^{n} X_{i}-n \mathrm{E}\left[X_{1}\right]}{n^{1 / p}} \rightarrow 0 \text { almost surely, as } n \rightarrow \infty
$$

is $\mathrm{E}\left[\left|X_{1}\right|^{p}\right]<\infty$. 
2. Let $\left\{X_{i}, i \geq 1\right\}$ be a sequence of positively associated random variables with finite variances. If

$$
\sum_{j=1}^{\infty} j^{-2} \operatorname{cov}\left(X_{j}, \sum_{i=1}^{j} X_{i}\right)<\infty
$$

then $\left(\sum_{i=1}^{n} X_{i}-\sum_{i=1}^{n} \mathrm{E}\left[X_{i}\right]\right) / n \rightarrow 0$ almost surely, as $n \rightarrow \infty$.

Theorem 3.2. Assume that $\mathrm{E}\left[\exp \left(-\delta T_{1}+\sigma \sqrt{T_{1}} \Phi^{-1}(U)\right)\right]<\infty$ and that $\mathrm{E}\left[\left|K_{1}\right|\right]<\infty$.

1. If $T_{l}, l=1, \ldots, n$, are negatively associated random variables, then (3.1) holds.

2. If, additionally, $K_{l}, l=1, \ldots, n$, are independently and identically distributed random variables, then (3.2) holds.

Proof. To prove part 1 , it suffices to show that, for any $u_{1}, u_{2} \in[0,1]$,

$$
\frac{1}{n} \sum_{i=1}^{n} f\left(T_{i}\right) \rightarrow F_{K_{1}}^{-1}\left(u_{2}\right) \mathrm{E}\left[\exp \left(-\delta T_{1}+\sigma \sqrt{T_{1}} \Phi^{-1}\left(u_{1}\right)\right)\right] \quad \text { almost surely, as } n \rightarrow \infty,
$$

where $f(x)=F_{K_{1}}^{-1}\left(u_{2}\right) \exp \left(-\delta x+\sigma \sqrt{x} \Phi^{-1}\left(u_{1}\right)\right)$. We can decompose $f(x)$ as $f(x)=$ $f_{1}(x)+f_{2}(x)$ with

$$
\begin{aligned}
f_{1}(x)= & F_{K_{i}}^{-1}\left(u_{2}\right) \exp \left(-\delta\left(\sqrt{x}-\frac{\sigma \Phi^{-1}\left(u_{1}\right)}{2 \delta}\right)^{2}+\frac{\sigma^{2}\left[\Phi^{-1}\left(u_{1}\right)\right]^{2}}{4 \delta}\right) 1\left\{x \leq\left[\frac{\sigma \Phi^{-1}\left(u_{1}\right)}{2 \delta}\right]^{2}\right\} \\
& +F_{K_{i}}^{-1}\left(u_{2}\right) \exp \left(\frac{\sigma^{2}\left[\Phi^{-1}\left(u_{1}\right)\right]^{2}}{4 \delta}\right) 1\left\{x>\left[\frac{\sigma \Phi^{-1}\left(u_{1}\right)}{2 \delta}\right]^{2}\right\}
\end{aligned}
$$

and

$$
\begin{aligned}
f_{2}(x)= & F_{K_{i}}^{-1}\left(u_{2}\right) \exp \left(-\delta\left(\sqrt{x}-\frac{\sigma \Phi^{-1}\left(u_{1}\right)}{2 \delta}\right)^{2}+\frac{\sigma^{2}\left[\Phi^{-1}\left(u_{1}\right)\right]^{2}}{4 \delta}\right) 1\left\{x>\left[\frac{\sigma \Phi^{-1}\left(u_{1}\right)}{2 \delta}\right]^{2}\right\} \\
& -F_{K_{i}}^{-1}\left(u_{2}\right) \exp \left(\frac{\sigma^{2}\left[\Phi^{-1}\left(u_{1}\right)\right]^{2}}{4 \delta}\right) 1\left\{x>\left[\frac{\sigma \Phi^{-1}\left(u_{1}\right)}{2 \delta}\right]^{2}\right\},
\end{aligned}
$$

where $1\{\cdot\}$ denotes the indicator function. Note that the sequences $\left\{f_{1}\left(T_{i}\right), i=1, \ldots, n\right\}$ and $\left\{f_{2}\left(T_{i}\right), i=1, \ldots, n\right\}$ are both negatively associated, since $f_{1}(x)$ and $f_{2}(x)$ are respectively increasing and decreasing in $x$. Hence, by part 1 of Lemma 3.3, we can show that

$$
\begin{aligned}
& \frac{1}{n} \sum_{i=1}^{n} f_{1}\left(T_{i}\right) \rightarrow F_{K_{i}}^{-1}\left(u_{2}\right) \mathrm{E}\left[\exp \left(-\delta\left(\sqrt{T_{1}}-\frac{\sigma \Phi^{-1}\left(u_{1}\right)}{2 \delta}\right)^{2}+\frac{\sigma^{2}\left[\Phi^{-1}\left(u_{1}\right)\right]^{2}}{4 \delta}\right)\right. \\
& \left.\times 1\left\{T_{1} \leq\left[\frac{\sigma \Phi^{-1}\left(u_{1}\right)}{2 \delta}\right]^{2}\right\}\right] \\
& +F_{K_{i}}^{-1}\left(u_{2}\right) \exp \left(\frac{\sigma^{2}\left[\Phi^{-1}\left(u_{1}\right)\right]^{2}}{4 \delta}\right) \mathrm{E}\left[1\left\{T_{1}>\left[\frac{\sigma \Phi^{-1}\left(u_{1}\right)}{2 \delta}\right]^{2}\right\}\right]
\end{aligned}
$$

almost surely, as $n \rightarrow \infty$, (3.4) 
and that

$$
\begin{aligned}
& \frac{1}{n} \sum_{i=1}^{n} f_{2}\left(T_{i}\right) \rightarrow F_{K_{i}}^{-1}\left(u_{2}\right) \mathrm{E} {\left[\exp \left(-\delta\left(\sqrt{T}_{1}-\frac{\sigma \Phi^{-1}\left(u_{1}\right)}{2 \delta}\right)^{2}+\frac{\sigma^{2}\left[\Phi^{-1}\left(u_{1}\right)\right]^{2}}{4 \delta}\right)\right.} \\
&\left.\times 1\left\{T_{1}>\left[\frac{\sigma \Phi^{-1}\left(u_{1}\right)}{2 \delta}\right]^{2}\right\}\right] \\
&-F_{K_{i}}^{-1}\left(u_{2}\right) \exp \left(\frac{\sigma^{2}\left[\Phi^{-1}\left(u_{1}\right)\right]^{2}}{4 \delta}\right) \mathrm{E}\left[1\left\{T_{1}>\left[\frac{\sigma \Phi^{-1}\left(u_{1}\right)}{2 \delta}\right]^{2}\right\}\right] \\
& \text { almost surely, as } n \rightarrow \infty .
\end{aligned}
$$

The combination of (3.4) and (3.5) immediately yields (3.3).

To prove part 2, we let

$$
Z_{i}=K_{i} \exp \left(-\delta T_{i}+\sigma \sqrt{T_{i}} \Phi^{-1}(U)\right) \text { and } V_{i}=\mathrm{E}\left[X_{i}\right] \exp \left(-\delta T_{i}+\sigma \sqrt{T_{i}} \Phi^{-1}(U)\right) .
$$

Note that $K_{l}, l=1, \ldots, n$, are independently and identically distributed random variables. Then, similarly to in the proof of part 1 of Theorem 3.1, we find that

$$
\frac{1}{n} \sum_{i=1}^{n}\left(Z_{i}-V_{i}\right) \rightarrow 0 \quad \text { almost surely, as } n \rightarrow \infty,
$$

from which it immediately follows that

$$
\frac{1}{n} \sum_{i=1}^{n} V_{i} \rightarrow \mu^{\mathrm{u}} \quad \text { almost surely, as } n \rightarrow \infty .
$$

The proof is thus complete.

Theorem 3.3. 1. Let $T_{j}, j=1, \ldots, n$, be positively associated random variables satisfying conditions (C1), (C2), and (C3) of Lemma 3.2. Furthermore, assume that $T_{j} \leq M$ for $j=$ $1,2, \ldots$, where $M$ is a constant, and that

$$
\sum_{j=1}^{\infty} j^{-2} \sum_{k=1}^{j}\left|\operatorname{cov}\left(T_{j}, T_{k}\right)\right|^{1 / 5}<\infty
$$

Then (3.1) holds.

2. If $K_{j}, j=1, \ldots, n$, are independently and identically distributed random variables, then (3.2) holds.

Proof. To prove part 1 it is sufficient to verify (3.3). We decompose the function $f(x)$ in (3.3) as $f(x)=f_{1}(x)+f_{2}(x)$ as in the proof of Theorem 3.2. Then, if we can prove (3.4) and (3.5), combining them immediately yields (3.3).

By the monotonicity of $f_{1}(x)$ and $f_{2}(x)$, we know that the sequences $\left\{f_{1}\left(T_{i}\right), i=1, \ldots, n\right\}$ and $\left\{f_{2}\left(T_{i}\right), i=1, \ldots, n\right\}$ are positively associated. Consequently, by part 2 of Lemma 3.3, a sufficient condition for (3.4) and (3.5) to hold is that

$$
\sum_{j=1}^{\infty} j^{-2} \operatorname{cov}\left(f_{i}\left(T_{j}\right), \sum_{k=1}^{j} f_{i}\left(T_{k}\right)\right)<\infty, \quad i=1,2 .
$$


Now, combining Lemma 3.1 and Lemma 3.2 yields

$$
\begin{aligned}
\operatorname{cov}( & \left.f_{i}\left(T_{j}\right), f_{i}\left(T_{k}\right)\right) \\
= & \int_{0}^{M} \int_{0}^{M} f_{i}\left(t_{1}\right) f_{i}\left(t_{2}\right)\left(p_{j k}\left(t_{1}, t_{2}\right)-p\left(t_{1}\right) p\left(t_{2}\right)\right) \mathrm{d} t_{1} \mathrm{~d} t_{2} \\
\leq & \int_{0}^{M} \int_{0}^{M} f_{i}\left(t_{1}\right) f_{i}\left(t_{2}\right) \mathrm{d} t_{1} \mathrm{~d} t_{2} \\
& \quad \times \frac{1}{4 \pi^{2}} \int_{-D}^{D} \int_{-D}^{D}\left|\varphi_{j k}(s, t)-\varphi(s) \varphi(t)\right| \mathrm{d} s \mathrm{~d} t+\frac{6 \sqrt{2} B(1+A)}{D} \\
\leq & M^{2}\left[F_{X_{1}}^{-1}\left(u_{2}\right)\right]^{2} \exp \left(\frac{\sigma^{2}\left[\Phi^{-1}\left(u_{1}\right)\right]^{2}}{2 \delta}\right)\left\{\frac{D^{4}}{4 \pi^{2}}\left|\operatorname{cov}\left(T_{j}, T_{k}\right)\right|+\frac{6 \sqrt{2} B(1+A)}{D}\right\} \\
\leq & c M^{2}\left|\operatorname{cov}\left(T_{j}, T_{k}\right)\right|^{1 / 5},
\end{aligned}
$$

where $c \equiv c\left(u_{1}, u_{2}\right)$ denotes a positive constant and the last inequality results from setting $D=\operatorname{cov}\left(T_{i}, T_{k}\right)^{-1 / 5}$. Consequently, (3.7) holds due to assumption (3.6).

The proof of part 2 is similar to the proof of part 2 of Theorem 3.2. We omit the details.

\section{References}

Ahcan, A., Darkiewicz, G., Goovaerts, M. and Hoedemakers, T. (2006). Computation of convex bounds for present value functions with random payments. J. Comput. Appl. Math. 186, 23-42.

Beekman, J. A. And Fuelling, C. P. (1993). One approach to dual randomness in life insurance. Scand. Actuarial J. 1993, 173-182.

Bellhouse, D. R. And Panjer, H. H. (1981). Stochastic modelling of interest rates with applications to life contingencies - Part II. J. Risk Insurance 48, 628-637.

Birkel, T. (1988). A note on the strong law of large numbers for positively dependent random variables. Statist. Prob. Lett. 7, 17-20.

BoyLE, P. P. (1976). Rates of return as random variables. J. Risk Insurance 43, 693-711.

DhaEnE, J. (1989). Stochastic interest rates and autoregressive integrated moving average processes. ASTIN Bull. 19, 131-138.

Dhaene, J. AND Goovaerts, M. J. (1996). Dependency of risks and stop-loss order. ASTIN Bull. 26, 201-212.

Dhaene, J. AND Goovaerts, M. J. (1997). On the dependency of risks in the individual life model. Insurance Math. Econom. 19, 243-253.

Dhaene, J. et al. (2002a). The concept of comonotonicity in actuarial science and finance: theory. Insurance Math. Econom. 31, 3-33.

DhAENE, J. et al. (2002b). The concept of comonotonicity in actuarial science and finance: application. Insurance Math. Econom. 31, 133-161.

Goovaerts, M. J. And Dhaene, J. (1999). Supermodular ordering and stochastic annuities. Insurance Math. Econom. 24, 281-290.

Goovaerts, M. J. AND Redant, R. (1999). On the distribution of IBNR reserves. Insurance Math. Econom. 25 , 1-9.

Goovaerts, M. J., Dhaene, J. AND De Schepper, A. (2000). Stochastic upper bounds for present value functions. J. Risk Insurance Theory 67, 1-14.

Hall, P. and Heyde, C. C. (1980). Martingale Limit Theory and Its Application. Academic Press, New York.

Hoedemakers, T., Darkiewicz, G., Dhaene, J. and Goovaerts, M. (2006). On the distribution of life annuities with stochastic interest rates. To appear in Insurance Math. Econom.

KaAs, R., Dhaene, J. And Goovaerts, M. J. (2000). Upper and lower bounds for sums of random variables. Insurance Math. Econom. 27, 151-168.

KAAs, R. et al. (2002). A simple geometric proof that comonotonic risks have the convex-largest sum. ASTIN Bull. 32, 71-80.

LIN, Z. (2003). Asymptotic normality of kernel estimates of a density function under association dependence. Acta Math. Sci. 23, 345-350.

LIU, J., GAN, S. AND CHEN, P. (1999). The Hájeck-Rènyi inequality for the NA random variables and its application. Statist. Prob. Lett. 43, 99-105. (Correction: 44, 210.)

Newman, C. M. (1980). Normal fluctuations and the FKG inequalities. Commun. Math. Phys. 74, 119-128. 
Norberg, R. (1990). Payment measures, interest and discounting. An axiomatic approach with applications to insurance. Scand. Actuarial J. 1990, 14-33.

Panjer, H. H. And Bellhouse, D. R. (1980). Stochastic modelling of interest rates with applications to life contingencies. J. Risk Insurance 47, 91-110.

Parker, G. (1994a). Stochastic analysis of a portfolio of endowment insurance policies. Scand. Actuarial J. 1994, 119-130.

PARKer, G. (1994b). Two stochastic approaches for discounting actuarial functions. ASTIN Bull. 24, 167-181.

PARKer, G. (1997). Stochastic analysis of the interaction between investment and insurance risks. N. Amer. Actuarial J. 1, 55-71.

Simon, S., Goovaerts, M. J. And Dhaene, J. (2000). An easy computable upper bound for the price of an arithmetic Asian option. Insurance Math. Econom. 26, 175-183.

Vyncke, D., Goovaerts, M. J. AND Dhaene, J. (2001). Convex upper and lower bounds for present value functions. Appl. Stoch. Models Business Industry 17, 149-164.

Wang, S. And Dhaene, J. (1998). Comonotonicity, correlation order and stop-loss premiums. Insurance Math. Econom. 22, 235-243.

Waters, H. R. (1978). The moments and distributions of actuarial functions. J. Inst. Actuaries 105, 61-75.

WiLkIE, A. D. (1976). The rate of interest as a stochastic process: theory and applications. In Trans. 20th Internat. Congress Actuaries, Vol. 1 (Tokyo, 1976), pp. 325-338. 